\title{
State Dependent Symplecticity of Symmetric Methods ${ }^{\star}$
}

\author{
Felice Iavernaro and Brigida Pace \\ Dipartimento di Matematica, Università di Bari, Italy \\ felix@dm.uniba.it, pace@dm.uniba.it
}

\begin{abstract}
Despite symmetric one-step methods applied to Hamiltonian dynamical systems fail in general to be symplectic, we show that symmetry implies, however, a relation which is close to symplecticity and that we called state dependent symplecticity. We introduce such definition for general maps and analyze it from an analytical viewpoint in one simpler case. Some numerical tests are instead reported as a support of this feature in relation with the good long time behaviour of the solutions generated by symmetric methods.
\end{abstract}

Keywords: Hamiltonian and Poisson systems, symplecticity, symmetric methods.

Subject Classification: 65P10, 65L05, 37M15.

\section{Introduction}

In this paper we link the property of symmetry of a one step numerical integrator applied to the Hamiltonian system

$$
\dot{y}=J \nabla H(y), \quad J=\left(\begin{array}{rr}
0 & -I \\
I & 0
\end{array}\right), \quad y=(p, q)^{T}, \quad H(p, q): \mathbb{R}^{m} \times \mathbb{R}^{m} \longrightarrow \mathbb{R},
$$

with $I$ the identity matrix, to a relation (satisfied by the map representing the method itself) called state dependent symplecticity (sd-symplecticity) which is close to the standard symplecticity property of symplectic integrators (we refer to [5] for the general theory on Hamiltonian problems). Although we do not report exhaustive theoretical results, we give some insights on how such feature in turn relies on the good stability properties shared by symmetric methods when applied to particular but important Hamiltonian systems in a neighborhood of an equilibrium point. In the following we assume that

$$
y_{1}=\phi_{h}\left(y_{0}\right)
$$

is a one-step method of order $p$ applied to the problem (1) with stepsize $h$ (we assume regularity of the transformation $\left.\phi_{h}\right)$.

\footnotetext{
* This work was supported by COFIN-PRIN 2004 (project "Metodi numerici e software matematico per le applicazioni").
} 
Definition 1. The one-step method (2) is called sd-symplectic if, when applied to the problem (1), its Jacobian matrix satisfies

$$
\left(\frac{\partial y_{1}}{\partial y_{0}}\right)^{T} \widehat{J}\left(y_{1},-h\right)\left(\frac{\partial y_{1}}{\partial y_{0}}\right)=\widehat{J}\left(y_{0}, h\right),
$$

where $\widehat{J}$ is a skew-symmetric nonsingular matrix for all $h \leq h_{0}$.

Although for our purposes we have attached property (3) to a numerical method, we may extend its applicability to any parametric transformation in the form (2), where $h$ stands for the parameter. Furthermore, one easily realizes that the matrix $\widehat{J}(y, h)$ approximates $J$ up to the order of the method: $\widehat{J}(y, h)=$ $J+O\left(h^{p}\right)$.

The dependence of relation (3) on the stepsize $h$ is both implicit (since $y_{1}$ depends on $h$ ) and explicit. In the particular cases where the explicit dependence is missing or where $\widehat{J}(\cdot, \gamma)$ is an even function of $\gamma$, we may recast (3) as

$$
\left(\frac{\partial y_{1}}{\partial y_{0}}\right)^{T} \widetilde{J}\left(y_{1}\right)\left(\frac{\partial y_{1}}{\partial y_{0}}\right)=\widetilde{J}\left(y_{0}\right),
$$

where $\widetilde{J}(y)=\widehat{J}(y, \pm h)$, and we are led back to the definition of a Poisson map with respect to the bracket

$$
\{F, G\}=\nabla F(y)^{T} \widetilde{J}(y) \nabla G(y), \quad \text { with } F, G: \mathbb{R}^{2 m} \rightarrow \mathbb{R} .
$$

Poisson systems generalize Hamiltonian systems in that they are defined by substituting to the matrix $J$ in (1) any skew-symmetric matrix $\widetilde{J}(y)$ satisfying the Jacobi identity $\{\{F, G\}, H\}+\{\{G, H\}, F\}+\{\{H, F\}, G\}=0$. The flow of a Poisson system

$$
\dot{z}=\widetilde{J}(z) \nabla H(z)
$$

is a Poisson map, and this justifies the study of Poisson integrators, that is numerical methods satisfying (4) when applied to (5).

Thus Definition 1 weakens the properties of a Poisson map. In general, neither the matrix $\widehat{J}(y, h)$ satisfies the Jacobi identity nor can it be stated that a sdsymplectic method is a Poisson integrator for a given set of almost Poisson problems. On the other hand, from a geometric viewpoint it seems like that property (3) may still imply, under suitable assumptions on the structure of the Hamiltonian and on the dynamics of the solution, an almost preservation of volumes of any bounded regions in the phase space, under the iterations of the method. This circumstance has already been detected and analysed in the simpler case of problems with one degree of freedom [4], where Definition 1 ] simplifies as $\mu\left(y_{1},-h\right)\left(\frac{\partial y_{1}}{\partial y_{0}}\right)^{T} J\left(\frac{\partial y_{1}}{\partial y_{0}}\right)=\mu\left(y_{0}, h\right) J$ with $\mu$ a scalar function. In this paper we consider the case of higher dimensional problems. For the special case of the trapezoidal method the computation simplifies remarkably and therefore we will use such simpler method to retrieve some theoretical results, while we will provide numerical evidence that other symmetric methods do exhibit similar behaviours. 


\section{Sd-Symplecticity of Symmetric Runge-Kutta Methods}

To see that any symmetric consistent RK method is sd-symplectic, we start the computation by considering the trapezoidal formula, which is the simplest symmetric non symplectic one step method. The application of the trapezoidal method to the Hamiltonian problem (11) defines the mapping

$$
y_{1}=y_{0}+\frac{h}{2} J\left(\nabla H\left(y_{0}\right)+\nabla H\left(y_{1}\right)\right) \text {. }
$$

By differentiating $y_{1}$ with respect to $y_{0}$, we get the variational equation

$$
\left(I-\frac{h}{2} J \nabla^{2} H\left(y_{1}\right)\right) \frac{\partial y_{1}}{\partial y_{0}}=\left(I+\frac{h}{2} J \nabla^{2} H\left(y_{0}\right)\right),
$$

where $\nabla^{2} H(y)$ is the Hessian matrix of $H(y)$. Setting $A^{ \pm}(y)=I \pm \frac{h}{2} J \nabla^{2} H(y)$ yields

$$
\left(\frac{\partial y_{1}}{\partial y_{0}}\right)^{T}\left(A^{-}\left(y_{1}\right)\right)^{T} J\left(A^{-}\left(y_{1}\right)\right) \frac{\partial y_{1}}{\partial y_{0}}=\left(A^{+}\left(y_{0}\right)\right)^{T} J\left(A^{+}\left(y_{0}\right)\right) .
$$

A direct computation shows that $\left(A^{-}(y)\right)^{T} J A^{-}(y)$ and $\left(A^{+}(y)\right)^{T} J A^{+}(y)$ define the same skew-symmetric matrix

$$
\widehat{J}(y, h)=J+\frac{h^{2}}{4} \nabla^{2} H(y) J \nabla^{2} H(y) .
$$

For $s$-stage symmetric RK methods, we follow a similar approach (see [4] for further details). In this case the mapping (2) reads

$$
y_{1}=y_{0}+h J\left(b^{T} \otimes I\right) \nabla H(K),
$$

where $K=\left[K_{1}^{T}, \ldots, K_{s}^{T}\right]^{T}$ is the block vector of the internal stages

$$
K=e \otimes y_{0}+h(A \otimes J) \nabla H(K),
$$

and $\nabla H(K) \equiv\left[\nabla^{T} H\left(K_{1}\right), \ldots, \nabla^{T} H\left(K_{s}\right)\right]^{T}$. Due to symmetry, we can split the term $\left(b^{T} \otimes I\right) \nabla H(K)$ of (7) in two (symmetric) terms depending uniquely on $y_{0}$ and $y_{1}$ respectively:

$$
y_{1}=y_{0}+\frac{h}{2} J\left(b^{T} \otimes I\right)\left[\nabla H\left(K^{+}\left(y_{0}\right)\right)+\nabla H\left(K^{-}\left(y_{1}\right)\right)\right],
$$

where

$$
K^{ \pm}(y)=e \otimes y \pm h(A \otimes J) \nabla H\left(K^{ \pm}(y)\right) .
$$

Differentiation of (8) with respect to $y_{0}$ yields a variational equation that looks similar to the one obtained for the trapezoidal method:

$$
\left(I-\frac{h}{2} J F_{-h}\left(y_{1}\right)\right) \frac{\partial y_{1}}{\partial y_{0}}=\left(I+\frac{h}{2} J F_{h}\left(y_{0}\right)\right),
$$


with

$$
F_{ \pm h}(y) \equiv\left(b^{T} \otimes I\right) \nabla^{2} H\left(K^{ \pm}(y)\right) \frac{\partial K^{ \pm}(y)}{\partial y}
$$

By defining

$$
\widehat{J}(y, \gamma)=\left(I+\frac{\gamma}{2} F_{\gamma}(y)\right)^{T} J\left(I+\frac{\gamma}{2} F_{\gamma}(y)\right),
$$

and exploiting symmetry, we finally arrive at (3).

\subsection{The Trapezoidal Method as a Simple Example}

The presence of the internal stages in a RK-method is responsible of the loss of symmetry of the matrix $\widehat{J}(y, \gamma)$ with respect to the second argument $\gamma$; in fact, in general, $F_{\gamma}(y) \neq F_{-\gamma}(y)$. Looking at (6) one realizes that for the trapezoidal method sd-simplecticity reduces to the standard preservation of a Poisson bracket structure (hereafter, to simplify the notation, we set again $\widetilde{J}(y) \equiv \widehat{J}(y, \pm h))$. This comes not as a surprise since the trapezoidal method is conjugate to the midpoint implicit method which is symplectic [3. Therefore the simpler condition (6) is well understood in terms of measure preserving properties 1 . In the phase space $\mathbb{R}^{2 m}$ consider a 2 -dimensional sub-manifold $\mathcal{M}$ obtained as the image of a compact set $K \subset \mathbb{R}^{2}$ through a continuously differentiable function $\psi:(s, t) \in K \mapsto(p, q) \in \mathcal{M}$, that is $\psi(K)=\mathcal{M}$. The scalar quantity

$$
\widehat{\Omega}(\mathcal{M})=\iint_{K}\left(\frac{\partial \psi}{\partial s}(s, t)\right)^{T} \widetilde{J}(\psi(s, t))\left(\frac{\partial \psi}{\partial t}(s, t)\right) d s d t .
$$

is the sum of the scaled oriented areas of the projections of $\mathcal{M}$ onto the orthogonal planes $\left(p_{i}, q_{i}\right), i=1, \ldots, m$. The term "scaled areas" means that $\widetilde{J}$ acts as a weight function. Therefore it turns out that the trapezoidal method preserves the quantity $\widehat{\Omega}(\mathcal{M})$, i.e.,

$$
\widehat{\Omega}\left(\phi_{h}(\mathcal{M})\right)=\widehat{\Omega}(\mathcal{M}),
$$

with $\widetilde{J}(\cdot)=\widehat{J}(\cdot, \pm h)$ defined in ([6). For $h \rightarrow 0$ we get $\widehat{J} \rightarrow J$ and (13) reduces to the classical geometrical interpretation of symplecticity.

From (3), for the trapezoidal method we get

$$
\begin{aligned}
\left(\frac{\partial y_{n}}{\partial y_{0}}\right)^{T} \widetilde{J}\left(y_{n}\right)\left(\frac{\partial y_{n}}{\partial y_{0}}\right) & =\left(\frac{\partial y_{n}}{\partial y_{n-1}} \frac{\partial y_{n-1}}{\partial y_{0}}\right)^{T} \widetilde{J}\left(y_{n}\right)\left(\frac{\partial y_{n}}{\partial y_{n-1}} \frac{\partial y_{n-1}}{\partial y_{0}}\right) \\
& =\left(\frac{\partial y_{n-1}}{\partial y_{0}}\right)^{T} \widetilde{J}\left(y_{n-1}\right)\left(\frac{\partial y_{n-1}}{\partial y_{0}}\right)
\end{aligned}
$$

and an induction process allows us to link the generic state vector $y_{n}$ to the initial one:

$$
\left(\frac{\partial y_{n}}{\partial y_{0}}\right)^{T} \widetilde{J}\left(y_{n}\right)\left(\frac{\partial y_{n}}{\partial y_{0}}\right)=\widetilde{J}\left(y_{0}\right)
$$

\footnotetext{
${ }^{1}$ As guide lines for the following description, we adopt the same argument and notations exploited in $[3$ to describe the geometric interpretation of symplecticity.
} 
A symplectic transformation is volume preserving. Analogously a Poisson transformation preserves a non-Euclidean measure (scaled volume). To find out the expression of the scaled volume preserved by the trapezoidal method, we consider the determinants of both sides of (14)

$$
\operatorname{det}\left(\frac{\partial y_{n}}{\partial y_{0}}\right)=\left(\frac{\operatorname{det}\left(\widetilde{J}\left(y_{0}\right)\right)}{\operatorname{det}\left(\widetilde{J}\left(y_{n}\right)\right)}\right)^{\frac{1}{2}}
$$

from which we obtain the following scaled volume conservation property:

$$
\text { scaled- } \operatorname{Vol}\left(S_{n}\right)=\operatorname{scaled}-\operatorname{Vol}\left(S_{0}\right),
$$

where the scaled volume of a $2 d$-dimensional region $S \subset \Omega$ defined by the trapezoidal method is

scaled-Vol $(S) \equiv \int_{S}\left(\operatorname{det}(\widetilde{J}(y))^{\frac{1}{2}} d y=\int_{S} \sqrt{\operatorname{det}\left(J+\frac{h^{2}}{4} \nabla^{2} H(y) J \nabla^{2} H(y)\right)} d y\right.$.

In particular, for separable Hamiltonian systems $H(p, q)=T(p)+U(q)$, (17) reduces to

$$
\text { scaled-Vol }(S)=\int_{S} \operatorname{det}\left(I+\frac{h^{2}}{4} U^{\prime \prime}(q) T^{\prime \prime}(p)\right) d p d q .
$$

The Taylor expansion of (15) yields

$$
\operatorname{det}\left(\frac{\partial y_{n}}{\partial y_{0}}\right)=1+O\left(h^{2}\right)
$$

where the $O\left(h^{2}\right)$ term may be assumed independent of $n$ if the Hessian matrix $\nabla^{2} H\left(y_{n}\right)$ remains bounded. This is true for systems (like the nonlinear pendulum) where the entries of the Hessian matrix are bounded functions or, more importantly, when the solution $y_{n}$ itself lies in a compact set of the phase space 2 . In such a case we obtain

$$
\int_{S_{n}} d y_{n}=\int_{S_{0}} d y_{0}+O\left(h^{2}\right),
$$

where the $O\left(h^{2}\right)$ term is independent of the integration time, which states a nearby-preservation property of the volumes.

Due to the appearance of the internal stages, it is not possible to retrieve a relation so easy as (14) for higher order RK methods. Consequently, the remainder in the analogue expression of (18) for a symmetric RK method, is expected

${ }^{2}$ This is a standard assumption when investigating the long time behaviour of the solutions of Hamiltonian systems (see for example [3, Theorem 8.1, page 312). 
to depend on the time $t_{n}$, but this is not always true. From (10) we obtain the analogue of (15) for symmetric RK methods:

$$
\operatorname{det}\left(\frac{\partial y_{n}}{\partial y_{0}}\right)=\frac{\prod_{i=0}^{n-1} \operatorname{det}\left(I+\frac{h}{2} J F_{h}\left(y_{i}\right)\right)}{\prod_{i=0}^{n-1} \operatorname{det}\left(I-\frac{h}{2} J F_{-h}\left(y_{i}\right)\right)}
$$

It turns out that the left hand side remains indeed bounded for the dynamics of many interesting Hamiltonian system. Such circumstance has been analysed for two-dimensional problems in [4], and is related to a global (rather than local) character of the solution. In the next section we report a few examples to give numerical evidence that the same may occur in higher dimensional systems.

\section{$3 \quad$ Numerical Results}

Hereafter we list four problems used for our tests together with a brief description (for further details see Chap. I of 3 and reference therein). All of them have separable Hamiltonian function in the form $H(p, q)=1 / 2 p^{T} p-U(q)$ (they come indeed from the application of Newton's second law), with the potential $U$ satisfying the symmetry relation $U(-q)=U(q)$. These conditions seem to be right ingredients that makes the determinants in (19) $O\left(h^{p}\right)$-bounded, independently of the time $t_{n} 3$

- TEST 1: Two-body Problem. The dynamics of two bodies attracted by their gravitational forces lies in a plane and it is identified by the (normalized) Hamiltonian function

$$
H\left(p_{1}, p_{2}, q_{1}, q_{2}\right)=\frac{1}{2}\left(p_{1}^{2}+p_{2}^{2}\right)-\frac{1}{\left(q_{1}^{2}+q_{2}^{2}\right)^{\frac{1}{2}}},
$$

where $p=\left(p_{1}, p_{2}\right)^{T}$ and $q=\left(q_{1}, q_{2}\right)^{T}$ are the velocity and position vectors of one body in a coordinate system centred at the second body.

- TEST 2: Perturbed two-body Problem. The same as the two body-problem with the addition of a perturbation term that accounts for non-Newtonian interactions:

$$
H\left(p_{1}, p_{2}, q_{1}, q_{2}\right)=\frac{1}{2}\left(p_{1}^{2}+p_{2}^{2}\right)-\frac{1}{\left(q_{1}^{2}+q_{2}^{2}\right)^{\frac{1}{2}}}-\frac{\mu}{\left(q_{1}^{2}+q_{2}^{2}\right)^{\frac{3}{2}}},
$$

where $|\mu|$ is a small real number (here set equal to $10^{-2}$ ).

- TEST 3: Fermi-Pasta-Ulam Problem. In the presented form, this problem describes the interaction of $2 m$ mass points linked with alternating soft nonlinear and stiff linear springs, in a one-dimensional lattice with fixed end points $\left(q_{0}=q_{2 m+1}=0\right)$. The Hamiltonian function is

$$
H(p, q)=\frac{1}{2} \sum_{i=1}^{m}\left(p_{2 i-1}^{2}+p_{2 i}^{2}\right)+\frac{\omega^{2}}{4} \sum_{i=1}^{m}\left(q_{2 i}+q_{2 i-1}\right)^{2}+\sum_{i=0}^{m}\left(q_{2 i+1}+q_{2 i}\right)^{4} .
$$

We chose $m=3$ (6 degrees of freedom) and $\omega=50$.

${ }^{3}$ Some counterexamples of reversible Hamiltonian systems with $U(-q) \neq U(q)$ for which symmetric non symplectic RK-methods are not appropriate can be found in [1]. 

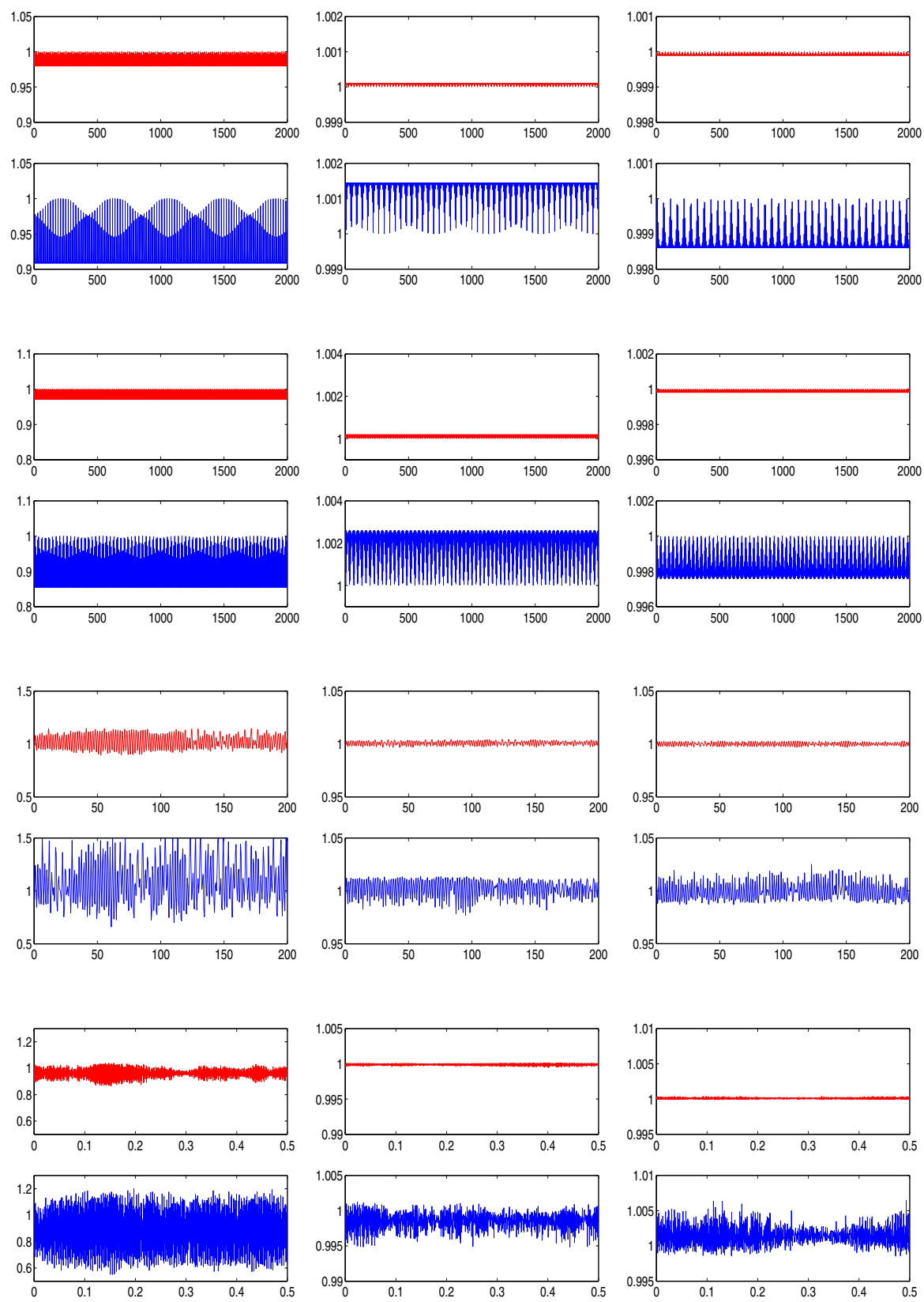

Fig. 1. Nearby volume preservation of the trapezoidal method (first column), Lobatto IIIA of order 4 (second column) and Lobatto IIIB of order 4 (right column). The $i$-th row displays the results for TEST $i$, for $i=1, \ldots 4$. Each figure consists of two pictures reporting the quantity defined in (19) of the methods applied with a given stepsize $h$ (upper plot) and $\bar{h}=2 h$ (lower plot). The list of the stepsizes $h$ is: TEST 1-2: $h=0.1$; TEST 3: $h=0.25 ;$ TEST $4: h=5 \cdot 10^{-5}$. 
- TEST 4: Molecular dynamics. Neutral atoms and molecules are subject to two distinct forces, one attracting and the other repelling, in the limit of large distance and short distance. This may be accounted for by considering pairpotentials like the Lennard-Jones potential (also known as the 6-12 potential) which, for the atoms $i$ and $j$ at a distance $r$, reads

$$
V_{i j}(r)=4 \varepsilon_{i j}\left(\left(\frac{\sigma_{i j}}{r}\right)^{12}-\left(\frac{\sigma_{i j}}{r}\right)^{6}\right) .
$$

The resulting system, simulating the dynamics of a network of $N$ particles, has Hamiltonian

$$
H(p, q)=\frac{1}{2} \sum_{i=1}^{N} \frac{1}{m_{i}} p_{i}^{T} p_{i}+\sum_{i=1}^{N} \sum_{j=i+1}^{N} V_{i j}\left(\left\|q_{i}-q_{j}\right\|\right) .
$$

In our experiment we have considered $N=7$ argon atoms $\left(m_{i}=m=\right.$ $66.34 \cdot 10^{-27} \mathrm{Kg}$ ) lying on a plane, with six equilibrium points located at the vertices of a regular hexagon and the remaining one at its centre, and

$$
\varepsilon_{i j}=\varepsilon \simeq 1.6540 \cdot 10^{-21} \mathrm{~J}, \quad \sigma_{i j}=\sigma=0.341 \cdot 10^{-9} \mathrm{~m}
$$

As initial conditions, we chose null velocities and positions slightly far away from the equilibria.

As numerical integrators we have used the LobattoIIIA and LobattoIIIB methods of order 4 and, for comparison purposes, the trapezoidal method. The related results have been displayed in the central, right and left columns of Figure 1 respectively. They report the quantity $\operatorname{det}\left(\partial y_{n} / \partial y_{0}\right)$ defined in (19) in correspondence of two different stepsizes in order to better infer its independence of the time integration interval. We have avoided to plot the residual $\left\|\left(\partial y_{n} / \partial y_{0}\right)^{T} J\left(\partial y_{n} / \partial y_{0}\right)-J\right\|$ since in general it fails to remain bounded even for the trapezoidal method and therefore it does not make sense.

\section{References}

1. E. Faou, E. Hairer and T.-L. Pham, Energy conservation with non-symplectic methods: examples and counter-examples, BIT Numerical Mathematics, 44 (2004), 699 709.

2. E. Hairer and C. Lubich, Symmetric multistep methods over long times, Numer. Math., 97 (2004), 699-723.

3. E. Hairer, C. Lubich, and G. Wanner, Geometric numerical integration. Structurepreserving algorithms for ordinary differential equations., Springer Series in Computational Mathematics, 31, Springer-Verlag, Berlin, 2002.

4. F. Iavernaro and D. Trigiante, State dependent symplecticity and area preserving numerical methods, (submitted).

5. K. R. Meyer, G. R. Hall, Introduction to Hamiltonian dynamical systems and the $N$-body problem, Applied Mathematical Sciences 90, Springer-Verlag, New York, 1992. 